\title{
Tabularia
}

TABULARIA Sources écrites des mondes normands médiévaux

\section{Une nouvelle source pour l'histoire du monastère de la Croix-Saint-Ouen à la fin du IXe siècle}

A new source towards the history of Croix-Saint-Ouen monastery at the end of the ninth century

Jacques Le Maho

\section{CpenEdition}

Journals

Édition électronique

URL : http://journals.openedition.org/tabularia/2485

DOI : $10.4000 /$ tabularia. 2485

ISSN : 1630-7364

Éditeur :

CRAHAM - Centre Michel de Boüard, Presses universitaires de Caen

Référence électronique

Jacques Le Maho, "Une nouvelle source pour l'histoire du monastère de la Croix-Saint-Ouen à la fin du IXe siècle », Tabularia [En ligne], Sources en ligne, mis en ligne le 10 mars 2005, consulté le 02 mai 2019. URL : http://journals.openedition.org/tabularia/2485; DOI : 10.4000/tabularia.2485 


\title{
Une nouvelle source pour l'histoire du monastère de la Croix-Saint-Ouen à la fin du IX ${ }^{\mathrm{e}}$ siècle
}

\section{A new source towards the history of Croix-Saint-Ouen monastery at the end of the ninth century}

\author{
Jacques LE MAHO (CNRS) \\ CRAHM-UMR 6577 \\ Université de Caen Basse-Normandie
}

\section{Résumé:}

Dans la lettre qu'il adresse entre 885 et 890 à Lambert, évêque du Mans, Gautier, évêque d'Orléans, demande à son correspondant de réserver le meilleur accueil à un groupe de moines qui fuient les Normands et s'apprêtent à quitter Orléans pour rejoindre le comté de Bayeux. Le premier éditeur de ce texte, Bernhard Bischoff, pensait pouvoir identifier ces moines à ceux de Saint-Ouen de Rouen, mais d'une nouvelle analyse, il ressort qu'il s'agit plus probablement de religieux de la Croix-Saint-Ouen, aujourd'hui La Croix-Saint-Leufroy (Eure). La lettre de Gautier permet ainsi d'en savoir un peu plus sur le sort de cette communauté entre 884, date de la confirmation des biens de sa mense conventuelle par le roi Carloman II, et 918, date de l'attribution par Charles le Simple des derniers restes de son patrimoine à l'abbaye de Saint-Germain-des-Prés.

Mots-clés: La Croix-Saint-Ouen, La Croix-Saint-Leufroy, Gautier, Lambert, Carloman, Charles le Simple, Normands, Paris, Thiverny, Bayeux.

\section{Abstract:}

In a letter written between 885 and 890 by Gautier, bishop of Orléans, to Lambert, bishop of Le Mans, Gautier asks his correspondent to greet favourably a party of monks fleeing the Northmen and about to leave Orléans to go back to the earldom of Bayeux. Bernhard Bischoff, the first to publish the text, thought these monks could be identified with those of Saint-Ouen in Rouen, but they are most probably friars from Croix-Saint-Ouen, today's La Croix-Saint-Leufroy in the Eure department. Gautier's letter thus enables us to know a little more about that community between 884, when king Carloman II confirmed what accrued to it through the monastery custom, and 918, when Charles the Simple bestowed the last remnants of his patrimony on Saint-Germain-des-Prés abbey.

Keywords: La Croix-Saint-Ouen, La Croix-Saint-Leufroy, Gautier, Lambert, Carloman, Charles the Simple, Normans, Paris, Thiverny, Bayeux.

Voici une vingtaine d'années, Bernhard Bischoff, éminent spécialiste allemand de la paléographie du Moyen Âge, publiait une série de huit lettres du dernier quart du IX ${ }^{\mathrm{e}}$ siècle, transcrites en notes tironiennes dans un manuscrit de la Bibliothèque 
nationale de France (BnF, nouv. acq. lat. 1096) ${ }^{1}$. Depuis lors, plusieurs historiens ont fait leur profit de ces textes qui recèlent nombre d'informations originales, notamment sur les conditions de l'accession au pouvoir du roi Eudes (888-898) et sur l'Église d'Orléans à la fin du IX ${ }^{\mathrm{e}}$ siècle $^{2}$. Toutefois, ces sources sont encore loin d'avoir été complètement exploitées.

$C^{\prime}$ 'est notamment le cas de la lettre $n^{\circ} 1$, transcrite au verso du folio $95^{3}$. Elle est adressée par G (autier), évêque d'Orléans (867/869-891) à un évêque L (ambert) identifié comme l'évêque du Mans (883-889/890). Dans cette missive, Gautier demande à son confrère de bien recevoir un groupe de moines qui, fuyant les Normands, avaient trouvé refuge auprès de lui à Orléans (dira Normannice persecutionis cogente crudelitate ad nostrae humilitatis confugere presidium); leurs vivres venant à manquer, ils s'apprêtent à rejoindre le comté de Bayeux où ils ont quelques possessions; pour cela, ils doivent passer par le diocèse du Mans (nunc ergo deficientibus eis victualibus sumptibus, per vestrum episcopatum Baiocensem comitatum, ubi rerum suarum aliqua habetur fiducia, in praesenti eos adire cogit necessitas). Les moines sont présentés comme des «monachi de C [..] sancti Audoeni», ce que Bernhard Bischoff propose, non sans hésitation car la formule d'abréviation du troisième mot, pour laquelle il suggère la lecture $\mathrm{Ctu}$, ne figure dans aucun répertoire de notes tironiennes, de développer en «monachi de conventu sancti Audoeni». Il s'agirait donc de moines de Saint-Ouen de Rouen, partis en exode lors de l'entrée des Normands dans la cité de la basse Seine en juillet $885^{4}$.

De prime abord, cette interprétation n'appelle pas d'objection particulière. Un départ des moines de Rouen pour le comté de Bayeux est parfaitement concevable dans la mesure où le diplôme délivré en 876 pour la confirmation des biens de SaintOuen fait mention de plusieurs domaines dans la partie occidentale de la province de Rouen. L'un d'eux au moins se trouve en Bessin, celui de Rausus, identifié à Rots, entre Caen et Bayeux 5 . Les faits se déroulant sans doute à l'époque du grand siège de Paris (885-887) et la région de Bayeux n'étant pas, à ce moment, menacée par les Normands, les moines de l'abbaye rouennaise auraient compté sur les ressources de ces propriétés périphériques pour assurer provisoirement leur subsistance.

1. BISCHOFF, Bernhard, «Briefe des neunten Jahrhunderts», in Anecdota novissima. Texte der vierten bis sechzehten Jahrhunderts, Bernhard BISCHOFF (éd.), Quellen und Untersuchungen zur lateinischen Philologie, 7, Stuttgart, A. Hiersemann, 1984, n 1, p. 126-135.

2. Guillot, Olivier, «Les étapes de l'accession d'Eudes au pouvoir royal», in Media in Francia... Recueil de Mélanges offert à Karl Ferdinand Werner, Maulévrier, Hérault-Éditions, 1989, p. 199-224; HEAD, Thomas, Hagiography and the cult of saints - The diocese of Orléans, 800-1200, Cambridge, Cambridge University Press, 1990.

3. Ibid., p. 126-127, texte $\mathrm{n}^{\circ} 1$.

4. Annales Vedastini, éd. Bernhard VON Simson, MGH., Scriptores rerum germanicarum in usum scholarum, 12, Hanovre, Hahn, 1909, p. 57.

5. Rots, Calvados, cant. Tilly-sur-Seulles; $c$. MUSSET, Lucien, « Ce qu'enseigne l'histoire d'un patrimoine monastique: Saint-Ouen de Rouen du IX ${ }^{\mathrm{e}}$ au XI ${ }^{\mathrm{e}}$ siècle», in Aspects de la société et de l'économie dans la Normandie médiévale ( $\mathrm{X}^{\mathrm{e}} \mathrm{XIII}{ }^{\mathrm{e}}$ siècles), Cahiers des Annales de Normandie $n^{\circ} 22$, Caen, 1988, p. 117. 
À y regarder de plus près, cependant, la lecture de Bernhard Bischoff soulève plusieurs questions. Comment se fait-il que nous retrouvions les moines de SaintOuen à Orléans, puis dans le Bessin, alors qu'il semble bien établi que cette communauté avait trouvé refuge dans son domaine de Condé-sur-Aisne, près de Soissons, et qu'à la suite des raids menés en 886 par le chef normand Siegfried dans le Soissonnais, elle s'était repliée avec une grande partie de ses reliques au sein de ses propriétés de Lotharingie, dans l'actuel Grand-Duché de Luxembourg ? Nous savons également qu'à cette époque, l'abbé de Saint-Ouen n'était autre que l'archevêque de Rouen et qu'il devait ordinairement résider dans son castrum de Braine, non loin de Condé 6 . Pourquoi ne trouve-t-on aucune allusion à ce haut personnage dans la lettre de l'évêque d'Orléans? N'est-il pas surprenant de voir la communauté rouennaise ainsi livrée à elle-même, quand on connait les liens étroits qui, jusqu'à sa transformation en établissement régulier dans la seconde moitié du Xe siècle, ont toujours uni cette maison au siège archiépiscopal?

Toutes ces difficultés sont levées si, de même que les noms de l'auteur de la lettre et de son destinataire sont indiqués par leurs seules initiales, on considère que l'abréviation $C[.$.$] correspond en fait aux trois premières lettres du nom du monastère.$ Il devient alors évident qu'il faut lire, non pas $\mathrm{Ctu}$, mais $\mathrm{Cru}$, ce qui donne «monachi de Cru (ce) sancti Audoeni». En réalité, il s'agirait donc des moines de l'abbaye de la Croix-Saint-Ouen, établissement qui fut fondé vers la fin du VII e siècle au diocèse d'Évreux, dans la vallée de l'Eure, sur le site de la commune actuelle de La CroixSaint-Leufroy ${ }^{7}$.

Depuis la découverte par Marcel Baudot de la copie d'un diplôme du roi Carloman II portant confirmation en 884 des biens de la mense conventuelle de la CroixSaint-Ouen, nous disposons de la liste des propriétés de ce monastère à la fin de l'époque carolingienne ${ }^{8}$. Or, si l'identification des toponymes pose des problèmes qui ne sont pas encore tous résolus, quatre noms se rapportent à des lieux situés in Autlina Sasonia, donc dans les parages du Bessin ou de l'Hiémois's, et sur ces quatre

6. Condé-sur-Aisne, Aisne, cant. Vailly-sur-Aisne; Braine, Aisne, chef-lieu de cant. Cf. MussET, Lucien, "Les translations de reliques en Normandie (IX ${ }^{\mathrm{e}}-\mathrm{XII}{ }^{\mathrm{e}}$ siècles) », in Les Saints dans la Normandie médiévale, Colloque de Cerisy-la-Salle (26-29 septembre 1996), Pierre BouET et François NEvEux (dir.), Caen, Presses universitaires de Caen, 2000, p. 101; du même auteur, voir également «Notes carolingiennes. III. Autour des domaines lotharingiens de Saint-Ouen de Rouen, Bulletin de la Société des antiquaires de Normandie, t. 57, 1963-1964, p. 385-391. Sur Braine, castrum des archevêques de Rouen, voir LAUER, Philippe, Le règne de Louis IV d'Outre-Mer, Paris, Librairie Émile Bouillon, 1900, p. 209.

7. La Croix-Saint-Leufroy, Eure, cant. Gaillon-Campagne. Les textes relatifs à la fondation ont fait l'objet d'une minutieuse étude critique de l'abbé Jean-Baptiste MESNEL, Les saints du diocèse d'Évreux, Évreux, Hérissey, $6^{\mathrm{e}}$ fascicule, 1918, Saint Leufroy abbé de la Croix, p. 1-147.

8. BAUDOT, Marcel, «L'abbaye de la Croix-Saint-Ouen à l'époque carolingienne, d'après le témoignage d'un diplôme de Carloman II », Bibliothèque de l'École des Chartes, t. CXLI, 1983, p. 5-21; sur le patrimoine de la Croix-Saint-Ouen et ses liens avec la marche bretonne, voir les remarques de BAUduIn, Pierre, La première Normandie ( $X^{e}-X I^{e}$ siècles), Caen, Presses Universitaires de Caen, 2004, p. 122-127.

9. Sur la question de l'Autlina (ou Autlingua) Saxonia et le problème de sa localisation, voir en dernier lieu BAUDOT, Marcel, «L'abbaye de la Croix.... », p. 13-16. 
domaines, deux au moins se trouvaient certainement dans le ressort du comté de Bayeux ${ }^{10}$. Sur ce point, rien ne s'oppose donc à ce que les réfugiés mentionnés dans la lettre aient effectivement appartenu au monastère de la Croix-Saint-Ouen.

Cette identification concorde aussi avec les indications que nous livre le diplôme de 884 sur la situation des moines de la Croix à la veille du siège de Paris par les Normands. L'essentiel de leur patrimoine était situé entre l'Eure et le nord-ouest du Parisis, zone directement exposée pendant toute cette période aux raids des Normands assurant le ravitaillement de l'armée de siège ; plusieurs de leurs domaines se trouvaient sur les bords de la Seine, dont Suresnes, aux portes de Paris ${ }^{11}$. Par suite d'un choix malheureux, c'est également dans le Bassin parisien, dans la vallée de l'Oise, que se trouvait alors le principal refuge des moines.

Tout semblait pourtant avoir été prévu. Dès 851 , les religieux avaient procédé en grande cérémonie, dans leur église abbatiale, à l'exhumation du corps de saint Leufroy, leur premier abbé, et à son transfert dans une châsse ; ainsi pourraient-ils l'emporter rapidement si une attaque normande les forçait à évacuer le monastère ${ }^{12}$. À la même époque, ils avaient obtenu du roi Charles le Chauve, grâce à une intervention de leur abbé Pardulus devenu évêque de Laon (848-856), la petite cella de Lamdas, dans le Perche drouais, pour leur servir de refuge (ad eorum confugium). Mais en raison de la menace grandissante dans l'espace compris entre la Loire moyenne et la Seine, l'évêque d'Évreux Gombert et l'archevêque de Rouen Vénilon obtinrent pour eux du même Charles le Chauve, entre 858 et 862 , un refuge considéré

10. Il s'agit de Malumhodum et de Burias, que Marcel Baudot propose d'identifier, soit à Malon et Buron, deux lieux-dits au nord-ouest de Caen, soit plutôt à Bures-les-Monts (dép. Calvados, cant. BényBocage) et Malloué (id.).

11. Suresnes, Hauts-de-Seine, chef-lieu de cant.; cf. BAUDOT, Marcel, «L'abbaye de la Croix... », p. 10-17.

12. La source est une notice du XII e siècle insérée à la suite de la Vita s. Leutfredi (Vita et miracula Leutfredi abbatis madriacensis, éd. Wilhem LEVISON, MGH, Scriptores, VII, p. 18). Elle procède apparemment d'un document original du IX ${ }^{\mathrm{e}}$ siècle retrouvé dans le reliquaire, mais comporte des modifications qui rendent parfois l'exposé des faits difficilement compréhensible. Il est notamment indiqué que les restes de l'abbé avaient été précédemment enterrés dans l'église abbatiale par crainte des Normands (de profundo hiatu terrae ubi posita fuerant propter metum Marcommanorum), alors que c'est le motif même pour lequel ils furent, en fait, exhumés : à titre de comparaison, voir le récit de la defossio des restes de saint Wandrille et de saint Ansbert dans l'abbatiale de Fontenelle au cours de ces mêmes années 850 (Inventio et miracula sancti Vulfranni, éd. Jean LAPORTE, Mélanges de la Société de l'Histoire de Normandie, Rouen-Paris, A. Lestringant-Auguste Picard, 1938, p. 25). Là où l'authentique carolingien devait notifier la translation de ces restes « in augustiorem locum», formule couramment employée en pareil cas, la notice du XII siècle parle d'un transfert « in loco celeberrimo », ce qui a été à l'origine de certaines erreurs d'interprétation. La plus fréquente consiste à identifier ce «lieu célèbre » à SaintGermain-des-Prés, ce qui signifierait, contre toute vraisemblance, que les moines de la Croix-SaintOuen s'étaient dessaisi des reliques de leur saint patron au profit d'une autre communauté, et qui plus est, sans que cette dernière fût nommément citée; il ressort d'ailleurs clairement du diplôme de Charles le Simple pour Saint-Germain-des-Prés que l'abbaye parisienne n'était en possession d'aucune des reliques de la Croix-Saint-Ouen avant 918 (cf. ci-dessous, note 42). La notice du XII siècle semble en revanche assez proche du texte de l'authentique carolingien lorsqu'elle précise que les restes de saint Leufroy furent placés dans un reliquaire (vasculo) et exposés dans l'église principale (ecclesia praecipua) du monastère de la Croix-Saint-Leufroy. 
comme plus sûr en raison de la proximité des palais royaux, la villa de Thiverny en Beauvaisis, sur la rive droite de l'Oise, en face de Creil ${ }^{13}$.

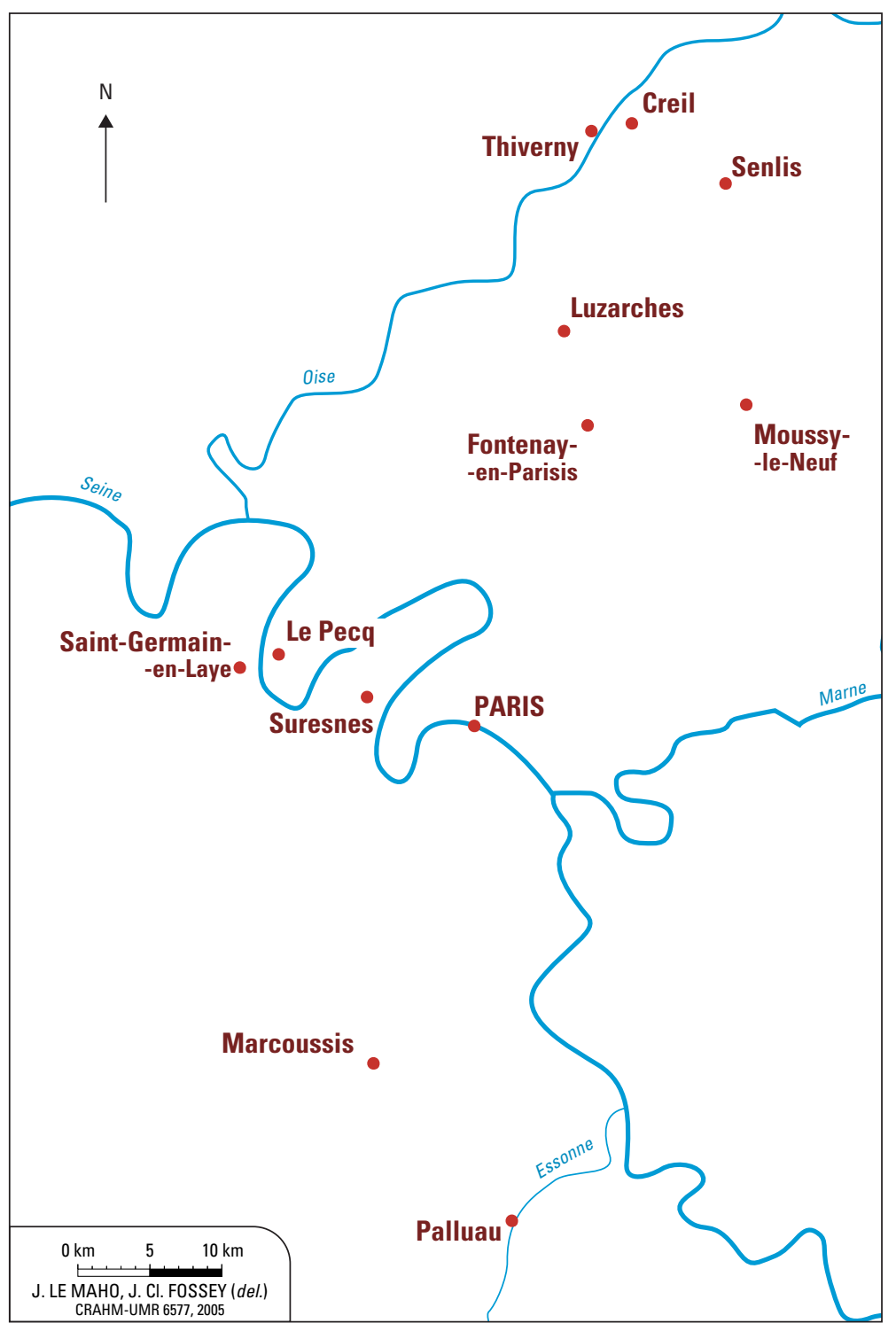

Fig. 1 : La région parisienne à la fin du $\mathrm{IX}^{\mathrm{e}}$ siècle, carte des lieux cités.

13. BAUDOT, Marcel, «L'abbaye de la Croix... », p. 16-18. 
C'est sans doute dans cette dernière propriété que les moines de la Croix se réfugièrent lors de l'attaque normande de 876 . Les annales de Saint-Bertin signalent l'entrée d'une flotte d'une centaine de navires dans la baie de Seine le 16 septembre; après avoir franchi sans difficulté l'obstacle du pont de Pîtres, les Normands demeurèrent dans le secteur de la Seine jusqu'au début de l'été 877 , pillant le pays en toute impunité ${ }^{14}$. Il convient peut-être aussi de situer à ce moment la fuite de l'évêque d'Évreux Sébar, épisode évoqué par Dudon de Saint-Quentin et généralement daté, jusqu'ici, des années 880 ou $890^{15}$. Ceci s'accorderait mieux avec les dates des deux seuls documents contemporains où apparaît le nom de Sébar, la liste des évêques présents au concile d'Attigny (870) et le procès-verbal de la visite des reliques de saint Ouen à Gasny par l'archevêque Riculphe (872-875) ${ }^{16}$. L'absence de Sébar pourrait également expliquer pourquoi, en 878, le comte de Poitiers Ermenon put s'emparer si facilement du castrum d'Évreux et utiliser cette place-forte pour piller la région ${ }^{17}$ : ce ne serait pas la première fois qu'à la suite d'un raid dévastateur des Normands sur une cité de Neustrie, un chef de guerre local aurait profité de la situation pour mettre à exécution ses projets de conquête territoriale ${ }^{18}$. Dans cette hypothèse, on peut penser que l'évêque Sébar et son clergé se replièrent dans un refuge proche de celui des moines de la Croix-Saint-Ouen. C'est du moins ce que suggère un petit groupe de dédicaces aux saints patrons de l'Église d'Évreux dans le secteur du fisc de Luzarches, à mi-chemin entre Paris et Thiverny ${ }^{19}$. Il faut sans doute rattacher aux mêmes événements le repli de l'archevêque de Rouen à Braine et celui des moines

14. Annales Bertiniani, éd. Georg WAITZ, MGH, Scriptores rerum germanicarum in usum scholarum, 5 , Hanovre, Hahn, 1883, p. 131-134; Annales Vedastini, éd. Bernhard von Simson, p. 41.

15. Dudon DE SAINT-QUENTIN, De gestis Normanniae ducum seu de moribus et actis primorum Normanniae ducum, éd. Jules LAIR, Mémoires de la Société des antiquaires de Normandie, t. XXIII, 1865, p. 157 ; récapitulation des hypothèses actuelles de datation par Pierre BAUDUIN, La première Normandie..., p. 165 , note 102 .

16. DuCHESNe, Louis, Fastes épiscopaux de l'ancienne Gaule, tome deuxième, L'Aquitaine et les Lyonnaises, deuxième édition, Paris, Fontemoing, 1910, p. 231 ; LAUER, Philippe, «Les translations des reliques de saint Ouen et de saint Leufroy du IX ${ }^{\mathrm{e}}$ au $\mathrm{X}^{\mathrm{e}}$ siècle et les deux abbayes de la Croix-Saint-Ouen », Bulletin philologique et historique (jusqu'à 1715) du Comité des travaux historiques et scientifiques, année 1921, 1923, p. 130, note 1.

17. Annales Bertiniani, éd. Georg WAITZ, p. 140.

18. On notera ainsi que l'attaque bretonne de 846 sur Bayeux, probablement menée par un certain Mangil, intervient un an après que les Annales de Saint-Bertin signalent une série d'attaques normandes le long des côtes de la Manche, de part et d'autre de la baie de Seine; en 846-847, il semble bien que l'évêque de Bayeux ne soit pas encore rentré dans sa cité (Annales Bertiniani, éd. Georg WAITZ, p. 32; Historia translationis corporum sanctorum Ragnoberti et Zenonis, Acta Sanctorum, 17 mai, 1680, tome 3, p. 615-624; sur Mangil, voir «Les premières annales de Fontenelle (Chronicon Fontanellense) », éd. dom Jean LAPORTE, Rouen-Paris, Société de l'histoire de Normandie, Mélanges-Documents, quinzième série, 1951, p. 79).

19. Luzarches, Val-d'Oise, chef-lieu de cant. Le séjour des reliques ébroïciennes a laissé plusieurs souvenirs dans ce secteur: un lieu-dit «Saint-Thaurin » à Luzarches (du nom du premier évêque d'Évreux); une tradition relative à la présence dans cette localité du corps de saint Éterne, évêque d’Évreux au VII e siècle (Gallia Christiana, t. XI, col. 567); la dédicace de l'église de Fontenay-en-Parisis à saint Aquilin, autre évêque d'Évreux (VIII ${ }^{\mathrm{e}}$ siècle). 
de Saint-Ouen à Condé. Le mouvement se poursuivit vers le début des années 880 avec l'installation probable des moines de l'abbaye de Fontenelle dans la région de Saint-Germain-en-Laye ${ }^{20}$. Quand, sans doute vers 885, l'Église de Sées eut obtenu à titre de refuge le domaine de Moussy-le-Neuf, sur la limite ouest du diocèse de Meaux $^{21}$, nombreuses étaient donc les communautés religieuses de la province de Rouen, et spécialement du secteur de la basse Seine, à avoir été transférées autour de Paris.

Cette situation aurait pu n'être que très temporaire. Cependant, rien n'indique un retour de l'archevêque de Rouen dans sa ville après le départ des Normands en 878. Il en est de même, comme on vient de le voir, pour l'évêque d'Évreux; la rupture que marquèrent, dans l'histoire de ce diocèse, les événements de la fin du règne de Charles le Chauve, semble confirmée par le fait que Sébar est le dernier nom sur la liste épiscopale carolingienne ${ }^{22}$. Dans ces conditions, on peut se demander si les moines de la Croix-Saint-Ouen ne se trouvaient pas encore à Thiverny durant l'été 884, lorsque leur fut délivré par le roi Carloman le diplôme de confirmation des biens de la mense conventuelle; l'acte est daté de Verberie, résidence royale située à faible distance de cette propriété du monastère. Il n'est pas exclu que les moines s'y soient encore trouvés à l'automne 885 , lorsque la flotte normande commença à remonter la Seine vers Paris, ce qui expliquerait leur décision de fuir vers Orléans.

À partir de ce moment, les événements se précipitèrent. En novembre, les Normands prirent et brûlèrent le castrum de Pontoise, à une trentaine de kilomètres en aval de Thiverny ${ }^{23}$. Les moines de l'abbaye de Fontenelle, qui devaient résider depuis le début des années 880 dans l'ouest parisien - où ils possédaient le vaste et riche domaine du Pecq sur la rive droite de la Seine, sans compter quelques possessions à Marcoussis dans le sud-est du Parisis et à Rivecourt sur l'Oise ${ }^{24}$ - eurent juste le temps de partir; prenant la direction du sud-ouest, ils arrivèrent à Chartres le 21 novembre, trois jours seulement avant l'arrivée de la flotte normande à Paris. Ils trouvèrent d'abord asile au monastère suburbain de Saint-Chéron, puis dans le secteur intramuros où, au mois de février 886 , une chapelle fut mise à leur disposition par l'évêque Aimon pour leurs reliques ${ }^{25}$. Les moines de Saint-Ouen ne tardèrent sans doute pas

20. Cf. ci-après, note 25 .

21. Moussy-le-Neuf, Seine-et-Marne, cant. Dammartin-en-Goëlle; sur l'origine de la propriété, voir les Miracula sanctae Opportunae, Acta Sanctorum, XII, avril, t. 3, p. 70; analyse de MusSET, Lucien, «L'exode des reliques du diocèse de Sées au temps des invasions normandes», Bulletin de la Société Historique et Archéologique de l'Orne, t. LXXXVIII, 1970, p. 10-11.

22. Duchesne, Louis, "Fastes épiscopaux... », p. 225.

23. Annales Vedastini, éd. Bernhard VON SiMSON, p. 57-58.

24. Le Pecq, dép. Yvelines, arr. Saint-Germain-en-Laye, chef-lieu de cant.; Marcoussis, Essonne, cant. Montlhéry; cf. LOT, Ferdinand, Études critiques sur l'abbaye de Saint-Wandrille, Paris, Champion, 1913, p. XXIII. Le repli des moines de Fontenelle dans la région parisienne est vraisemblablement consécutif à la grande offensive normande du début des années 880 entre la Somme et l'Escaut, qui chassa un grand nombre de communautés vers le sud (Annales Vedastini, éd. Bernhard VON SIMSON, p. 48).

25. Miracula sancti Wandregisili, Acta Sanctorum, juillet, t. V, p. 289; commentaires de LoT, Ferdinand, Études..., p. XXXIX. 
à évacuer, à leur tour, leur cella de Condé-sur-Aisne. En effet, prenant au dépourvu tous ceux qui avaient cru que l'attaque se limiterait, comme les fois précédentes, aux abords immédiats de la ville, l'onde d'insécurité s'étendit rapidement à un large rayon autour de Paris. C'est probablement dans son domaine épiscopal de Moussy-le-Neuf que l'évêque de Sées Adelhelm fut fait prisonnier avant d'être emmené hors de Francie pour être vendu sur un marché d'esclaves, comme il allait lui-même le raconter plus tard dans son livre des Miracles de sainte Opportune ${ }^{26}$.

$\mathrm{Si}$, comme tout porte à le croire, les moines de la Croix-Saint-Ouen sont bien les monachi évoqués dans la lettre de l'évêque d'Orléans, leur réflexe fut donc le même que ceux des moines de Fontenelle: partir vers le sud et chercher refuge dans une cité épiscopale. Ce repli vers les villes était un phénomène relativement nouveau. Au cours des quarante années qui s'étaient écoulées depuis la première incursion des Vikings dans la vallée de la Seine (en 841), les communautés monastiques avaient généralement cherché le salut dans la fuite vers leurs terres les plus éloignées. Mais, comme l'a bien montré Fernand Vercauteren, les années 880 marquèrent un tournant dans la politique de défense en Francie ${ }^{27}$. Les initiatives locales se multiplièrent et, un peu partout, les évêques et les comtes s'employèrent à réparer ou à renforcer les enceintes urbaines. À partir du milieu de la décennie, les cités épiscopales furent de plus en plus nombreuses à accueillir la population des monastères des faubourgs ou, comme dans le cas qui nous occupe ici, à offrir un refuge provisoire à des groupes de religieux en fuite.

En l'occurrence, le contenu de la lettre de Gautier s'accorde donc avec le témoignage d'Adrevald de Fleury qui, à la fin du IX ${ }^{\mathrm{e}}$ siècle, attribue à ce même évêque le mérite d'avoir restauré les murailles d'Orléans, offrant ainsi un abri à la population ${ }^{28}$. Dans les Miracula sancti Benedicti, Adrevald nous parle également de l'église SaintBenoît d'Orléans où se réfugièrent les moines de Fleury-sur-Loire au temps des invasions normandes ${ }^{29}$. À ces deux témoignages s'ajoute celui d'un acte de l'abbaye SaintMesmin de Micy, proche de la cité d'Orléans. Ce document est reconnu pour être un faux du XIe siècle, mais il rapporte une tradition non dénuée de vraisemblance, selon laquelle l' «Alleu de Saint-Mesmin », dans la zone intra-muros d'Orléans, aurait été à la même époque le refuge des moines de l'abbaye ${ }^{30}$.

26. Liber miraculorum sancte Opportune, Acta Sanctorum, April, III, p. 68; le récit d'Adelhelm a été traduit et commenté par Lucien MussET dans les Documents de l'Histoire de la Normandie, Michel DE BOÜARD (dir.), Toulouse, Privat, 1972, p. 70-71.

27. VerCAUteren, Fernand, «Comment s'est-on défendu, au IX siècle, dans l'Empire franc contre les invasions normandes? », Annales du XXXe congrès de la Fédération archéologique et historique de Belgique (1935), Bruxelles, Impr. De Meester, 1936, p. 117-132.

28. «Donec venerabilis pontifex eiusdem urbis, cum summa dicendus reverentia, Galterius, Deo inspirante, muros, per cuncta fere destructos civitatis, sagacitate nobilis ingenii qua praepollere cognoscitur restaurans, defensioni coaptaret populorum» (Miracula sancti Benedicti-Les miracles de saint Benoît, éd. Eugène DE CerTaIn, Paris, Société de l'Histoire de France nº 85, J. Renouard, 1858, p. 79).

29. Miracula sancti Benedicti, p. 78-79.

30. Tessien, Georges, Recueil des actes de Charles II le Chauve, roi de France, Académie des Inscriptions et Belles-Lettres, t. 2, Paris, Imprimerie nationale, 1952, p. 666, $n^{\circ} 504$. 
En précisant que leurs vivres étaient venus à manquer, ce qui obligeait les moines de la Croix-Saint-Ouen à repartir, la lettre de Gautier montre combien était difficile la tâche des évêques dans leur mission d'assistance aux réfugiés. Au sein d'une ville sans doute déjà surpeuplée, la prise en charge d'une communauté monastique venue d'un autre diocèse, coupée de ses bases et ne disposant donc, dans l'immédiat, d'aucune ressource, était un souci supplémentaire pour l'évêque d'Orléans. Malgré cela, il semble avoir tout fait pour offrir le meilleur accueil à ces religieux. Les moines de la Croix s'étaient sans doute recommandés des liens de confraternité unissant leur communauté à l'Église d'Orléans depuis le temps de Robert-le-Fort († 866), ce dernier ayant été abbé de leur établissement et, en même temps, de Saint-Aignan d'Orléans ${ }^{31}$. En usant de son droit de réquisition sur les loca deserta et sur les bâtiments du domaine public ou, plus simplement, en leur offrant l'asile de son palais épiscopal comme le fit l'évêque de Chartres pour les communautés de Fontenelle et de Saint-Chéron ${ }^{32}$, Gautier dut faire son possible pour trouver à ces moines un hébergement provisoire dans la cité. Il fit également du mieux qu'il put pour leur accorder quelques secours (prout contulit possibilitas, aliquantulum largiti sumus supplementum). Néanmoins, il était sûrement hors de question que leur frais de subsistance fussent durablement à la charge de son évêché.

Comme leur abbé n'est nulle part mentionné, il faut croire que, dans le sauvequi-peut général, les moines de la Croix-Saint-Ouen avaient été momentanément abandonnés à leur sort. S'il n'est nullement certain que l'abbé Willelmus mentionné dans l'acte de 884 soit identifiable à Guillaume le Pieux, futur duc d'Aquitaine, comme le pensait Marcel Baudot ${ }^{33}$, on peut toutefois supposer que le monastère de la Croix était alors sous la direction d'un abbé laïque ou, du moins, que l'abbé ne résidait pas en permanence dans cet établissement. Quoi qu'il en soit, il resterait à savoir pourquoi, alors que c'eût été son devoir en pareille circonstance, l'abbé n'avait pas proposé aux moines un domaine de la mense abbatiale ou l'une de ses propriétés personnelles pour leur servir de refuge. Le fait est difficilement explicable si l'on s'en tient à l'hypothèse de Guillaume le Pieux, car ce personnage disposait d'un immense patrimoine foncier dans des régions particulièrement sûres comme l'Auvergne, le Limousin ou le Maconnais ${ }^{34}$. On est ainsi conduit à se demander s'il ne s'agissait pas d'un autre Guillaume, possessionné dans le même secteur géographique que les moines

31. Sur les liens du monastère de la Croix-Saint-Ouen avec les Robertiens, cf. BAUDUIN, La première Normandie..., p. 122-128. Dans une lettre de 888, Gautier, évêque d’Orléans, reconnaît le roi Eudes et Robert de Neustrie (fils de Robert le Fort) comme ses seniores (BISCHOFF, Bernhard, «Briefe des Neunten Jahrhunderts », p. 135).

32. Voir ci-dessus, note 25.

33. Cf. sur ce point Bauduin, La première Normandie..., p. 123.

34. Laurenson-Rosaz, Charles, L’Auvergne et ses marges (Velay, Gévaudan) du VIII ${ }^{e}$ au XIe siècle, Les Cahiers de la Haute-Loire, Le Puy-en-Velay, 1987, p. 66-72; sur les reliques de Senlis, voir BIANCHINA, Nicole, "Saint-Frambourg de Senlis - Étude historique et archéologique », Revue Archéologique de l'Oise n²0, 1980, p. 5-16; voir également Musset, Lucien «Un curieux épisode de l'histoire ecclésiastique du Pays d'Auge au XI ${ }^{\text {e }}$ siècle : Equemauvile et les reliques de saint Vigor ", Annuaire des cinq départements de la Normandie, t. 145, 1987 (paru 1988), p. 93. 
et n'ayant donc pas les moyens de leur procurer, dans l'immédiat, un refuge offrant toute garantie de sécurité; nous verrons, un peu plus loin, un élément qui semble venir à l'appui de cette seconde hypothèse.

Les informations que nous livre la lettre de Gautier sur l'étape suivante de l'exode des moines - leur repli vers le comté de Bayeux, en passant par le Maine - sont d'un intérêt tout particulier en raison du rôle-clé de ces deux régions au sein de la marche «normanno-bretonne» de la seconde moitié du IX siècle $^{35}$. Le texte montre également l'importance, dans ce dispositif, du réseau de communications épistolaires qui, depuis le très haut Moyen Âge, reliait entre eux les évêchés de Neustrie. En l'occurrence, on constate que de tels documents pouvaient tenir lieu de sauf-conduits pour les groupes de religieux dans leurs déplacements. Les moines de la Croix ne furent pas les seuls à obtenir de Gautier une lettre de recommandation. Parmi les lettres publiées par Bernhard Bischoff, il en est une du même type que cet évêque écrivit pour «l'un de ses moines» qui, ne souhaitant pas rester plus longtemps dans ce pays ravagé par la "persécution cruelle des Païens ", avait décidé de partir en pèlerinage à Rome $^{36}$. Enfin, on pourra comparer l'itinéraire des moines de la Croix à celui qui fut suivi en sens inverse par les clercs de l'Église de Bayeux lorsqu'au début des années 920, ainsi que le rapporte la Translation de saint Magloire, ils s'enfuirent de leur diocèse pour venir se réfugier dans la région parisienne ${ }^{37}$. À cet épisode se rattache un lot de reliques bayeusaines déposé au $\mathrm{X}^{\mathrm{e}}$ siècle dans le trésor de la collégiale Saint-Frambourg de Senlis; fait significatif, il s'y trouve associé à un groupe de reliques du Maine, dont celles de saint Fraimbault, alias Frambourg, primitivement honoré à Saint-Fraimbaultde-Prières, près de Mayenne ${ }^{38}$. Lors de la même translation, les clercs de Bayeux rencontrèrent en chemin un groupe de confrères de l'église de Dol qui faisaient également route vers Paris; quand, aux environs de 930, la fin de la menace normande permit à ces derniers de rentrer chez eux, c'est par Orléans qu'ils regagnèrent la Bretagne ${ }^{39}$. La route méridionale que s'apprêtaient à suivre les moines de la Croix-Saint-Ouen devait donc être considérée comme un des itinéraires les plus sûrs. Située à peu près à égale distance des estuaires de la Seine et de la Loire, elle traversait des régions intérieures qui, de fait, furent rarement atteintes par les Normands au cours de leurs expéditions en Francie.

À supposer que les moines de la Croix-Saint-Ouen aient réussi à rejoindre sans encombre leurs domaines du comté de Bayeux, qu'advint-il d'eux par la suite? A priori, le choix de cette destination n'était peut-être pas très heureux, du moins si l'on en juge par le témoignage de Dudon de Saint-Quentin évoquant un raid des Normands sur Bayeux au cours du siège de Paris $(885-887)^{40}$. L'authenticité des faits

35. Sur cette question, voir BAUDUIN, Pierre, La première Normandie..., p. 14-122.

36. BISCHOFF, Bernhard, "Briefe des neunten Jahrhunderts ", p. 128-129, lettre n 3.

37. Guillotel, Hubert, «L'exode du clergé breton devant les invasions scandinaves », Mémoires de la Société d'Histoire et d'Archéologie de Bretagne, tome LIX-1982, p. 269-315.

38. Saint-Fraimbault-de-Prières, dép. Mayenne, cant. Mayenne-Est.

39. Guillotel, Hubert, "L’exode du clergé breton... », p. 294-295.

40. DUdOn DE SAINT-QUENTIN, «De gestis Normanniae ducum... », p. 157. 


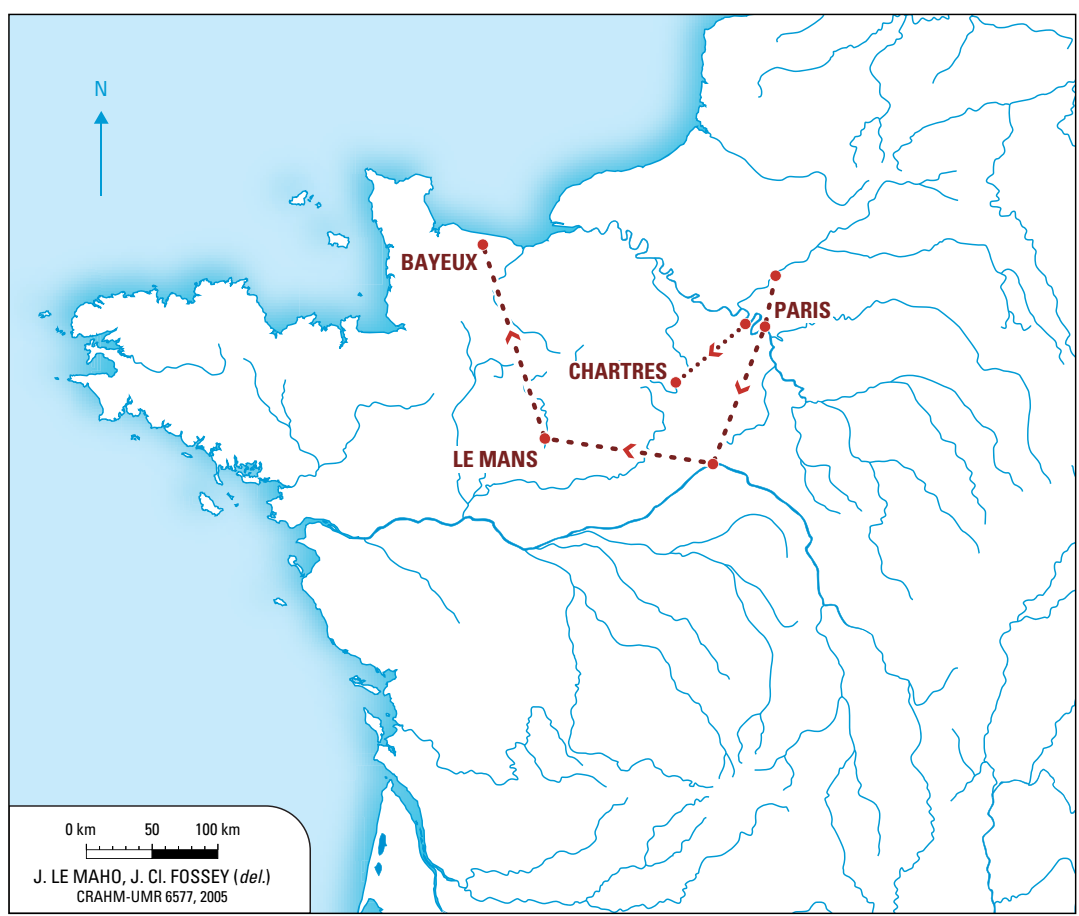

Fig. 2 : Exodes des moines de Fontenelle et de la Croix-Saint-Ouen au cours du siège de Paris (885-887).

rapportés par l'historiographe des premiers ducs nous paraît cependant loin d'être assurée, aussi bien en ce qui concerne la date de l'événement que le rôle de Rollon et la capture de la fille du comte de Bayeux au cours de ce raid. De même, nul ne sait au juste sur quel élément se fonde Mabillon lorsqu'il signale que le monastère de la Croix était encore en activité en $898^{41}$; il est à craindre que cette date n'ait pas d'autre justification que sa correspondance avec l'avènement de Charles le Simple, souverain dont le nom reste attaché à l'histoire des derniers temps de l'abbaye, en tant que liquidateur des biens de l'établissement. Le seul fait à peu près certain est que la communauté s'éteignit ou fut dissoute avant 918, car dans le diplôme du roi Charles notifiant la cession à Saint-Germain-des-Prés de la partie française du patrimoine de la CroixSaint-Ouen, il est précisé que les corps saints du monastère sont privés depuis longtemps des honneurs de l'office divin (membra sanctorum diu officio divino carentium); en contrepartie de sa donation, le roi charge les moines de Saint-Germain de rétablir et de perpétuer le culte de ces reliques en déshérence ${ }^{42}$.

41. Mabillon, Annales ordinis S. Benedicti, t. III, rééd. 1950, Macon, Protat, p. 302; cité par BaUdUIN, Pierre, La première Normandie..., p. 122.

42. LAUER, Philippe, Recueil des actes de Charles III le Simple, roi de France (893-923), Paris, Académie des Inscriptions et Belles-Lettres, t. 1, 1940, n 92, p. 211. 
Puisque la communauté semble ainsi s'évanouir à la fin du IXe ou au début du $\mathrm{X}^{\mathrm{e}}$ siècle, sa disparition n'est-elle pas précisément liée à l'exode au comté de Bayeux? A priori, on serait tenté de penser que les moines ne restèrent pas longtemps, en fait, dans cette partie occidentale de la province de Rouen. Le pays demeura relativement sûr jusqu'à l'hiver 889/890, mais pour autant qu'on puisse en juger par le diplôme de Carloman, leurs possessions n'y étaient ni très nombreuses, ni très importantes. À partir de la fin des années 880, comme nous avons tenté de le montrer dans plusieurs de nos travaux ${ }^{43}$, le secteur de la basse Seine fut provisoirement mis en sécurité grâce aux efforts de l'administration royale, qui fit de la cité de Rouen une grande place-refuge ; il en fut de même de la région parisienne, alors débarrassée pour plusieurs années de la menace normande. Fait significatif, c'est en direction de Rouen puis de Paris que se dirigèrent la plupart des communautés du Bessin et du Cotentin lorsqu'à la fin de l'année 889, elles furent chassées par les Normands venus mettre le siège devant le castrum de Saint-Lô ${ }^{44}$. Dans ces conditions, il n'y aurait rien d'étonnant à ce que les moines de la Croix aient regagné assez tôt le secteur de l'Oise et de l'ouest parisien, où ils avaient une grande partie de leurs propriétés et où, selon toute vraisemblance, ils résidaient déjà depuis plusieurs années lorsque les évènements de la fin 885 les avaient obligés à fuir vers Orléans.

Ceci rejoindrait une hypothèse de Marcel Baudot, qui se demandait si le domaine à l'origine de la commune actuelle de Lacroix-Saint-Ouen (sic), sur la rive gauche de l'Oise, près de Compiègne, n'était pas devenu pour quelque temps, après 884 , le siège de notre monastère ${ }^{45}$. Outre l'homonymie, on relève en effet plusieurs faits troublants, comme la présence de reliques de saint Ouen dans un caveau sous le chœur de l'église ${ }^{46}$. Il serait tentant de faire le rapprochement avec la mention des «saintes cendres de l'archevêque Ouen » dans l'acte de 918 pour Saint-Germain-des-Prés, en tête de la liste des reliques du monastère de la Croix ${ }^{47}$. À cette localité de l'Oise

43. LE MAHO, Jacques, «Rouen à l'époque des incursions vikings (841-911)», Bulletin de la Commission des Antiquités de la Seine-Maritime, t. 42, 1994 (paru 1995), p. 143-202; ID., "The fate of the ports of the lower Seine Valley at the end of the ninth century", Markets in early medieval Europe - Trading and 'productive' sites, 650-850, Tim PesTell and Katharina UlmsCHNEIDER (éd.), Wingather Press, Bollington, 2003, p. 234 - 247; ID., «Les premières installations normandes dans la basse vallée de la Seine (fin du IX ${ }^{\mathrm{e}}$ siècle) », in La progression des Vikings, des raids à la colonisation, Anne-Marie Flambard-Héricher (éd.), Publications de l'Université de Rouen, Cahiers du G.R.H.I.S n 14, 2003, p. 153-169.

44. LE MAHO, Jacques, «Un exode de reliques dans les pays de la basse Seine à la fin du IX $\mathrm{IX}^{\mathrm{e}}$ siècle», Bulletin de la Commission Départementale des Antiquités de la Seine-Maritime, t. XLVI, 1998 (paru 1999), p. 136-188.

45. Lacroix-Saint-Ouen, dép. Oise, cant. Compiègne Sud-Est; BAudot Marcel, «l'abbaye de la CroixSaint-Ouen...", p. 18.

46. Nous remercions Jean-Claude Malsy de nous avoir communiqué les éléments inédits de sa notice sur Lacroix-Saint-Ouen, à paraître dans le nouveau Dictionnaire des noms de lieux de l'Oise.

47. LAUER, Philippe, «Les translations des reliques... », p. 211. Signalons toutefois une autre hypothèse de Jean-Claude Malsy pour expliquer la présence de ces reliques: les moines de Saint-Ouen de Rouen auraient fait étape à Crux lors de la translation des restes de leur saint patron à Condé-sur-Aisne ( $c f$. note précédente). Il paraît en tout cas certain que l'église de Crux abritait seulement quelques fragments, 
s'attache également une tradition calquée sur la légende fondatrice du monastère du diocèse d'Évreux : l'église du lieu aurait été fondée par saint Ouen en personne, à la suite de la découverte par ce prélat, au cours d'une halte dans la forêt de Compiègne, d'une croix de neige ${ }^{48}$. On pourra certes objecter que ce domaine ne figure ni dans le diplôme de Carloman II de 884, ni dans celui de Charles le Simple de 918, et qu'en revanche, il est toujours mentionné à partir du XII ${ }^{e}$ siècle comme prieuré de Saint-Médard de Soissons ${ }^{49}$. Si l'on part de l'hypothèse qu'il relevait primitivement de la mense abbatiale, ces difficultés ne sont cependant qu'apparentes. Dans ce cas, en effet, il est normal que le domaine n'apparaisse pas dans les inventaires de la mense conventuelle et qu'il ait connu un destin différent lors de la partition des biens de l'abbaye au début du $\mathrm{X}^{\mathrm{e}}$ siècle $\mathrm{e}^{50}$. Ajoutons que ce domaine était voisin du palais de Verberie où fut délivré le diplôme du 16 août 884 , à la demande et en présence de Guillaume, abbé de la Croix-Saint-Ouen. Il resterait à savoir pourquoi le siège du monastère fut installé sur ce site plutôt que dans la villa de Thiverny, alors que cette dernière avait été choisie dès le début des années 860 comme base de repli pour les moines en cas d'attaque normande. Un élément de réponse pourrait se trouver dans la présence d'une fortification, ce qui, compte tenu de la typologie des castella de ce secteur de la Neustrie à la fin de l'époque carolingienne, justifierait la situation du prieuré de Saint-Médard (aujourd'hui disparu) dans la zone marécageuse des bords de l'Oise ${ }^{51}$.

48. la majeure partie du corps de saint Ouen demeurant en possession des religieux de l'abbaye rouennaise; il n'y a donc pas lieu de remettre en cause la tradition qui fait venir de Condé-sur-Aisne les restes de saint Ouen lors de leur rapatriement à Rouen en 918.

48. Légende insérée au cartularium antiquum de Saint-Médard, sous la forme d'un diplôme de Dagobert (DefFente, Denis (éd.), Saint-Médard, Trésors d’une abbaye royale, Éditions Somogy, Paris, 1996, p. 86). À la Croix-Saint-Ouen au diocèse d'Évreux, c'est une croix lumineuse qui serait apparue à saint Ouen (Vita IIa S. Audoeni, Acta Sanctorum, Aug. IV, p. 816).

49. Des actes de Saint-Médard citent l'abbati (ol) am de Cruce Sancti Audoeni parmi les biens confirmés à ce monastère par le pape Eugène II, par Charles le Chauve et par le roi Eudes, mais ils sont depuis longtemps reconnus comme des faux, réalisés entre le X ${ }^{\mathrm{e}}$ et le XII ${ }^{\mathrm{e}}$ siècle (TeSsIER, Georges, Recueil des actes de Charles II le Chauve..., t. 2, n 493, p. 636; DefFente, Denis, Saint-Médard..., p. 84-85 et 94).

50. Un passage des Gesta de Dudon de Saint-Quentin pourrait laisser supposer que le vicus de Crux, près de Compiègne, fut un moment réuni au domaine royal: vers 943, Louis IV d'Outremer aurait invité Hugues le Grand à venir l'y rencontrer (DUDON DE SAINT-QUENTIN, « De gestis Normanniae ducum... », p. 234; commentaire de LAUER, Philippe, Le règne de Louis IV d'Outre-Mer, Paris, Honoré Champion, 1900, p. 118, note 3). Cependant, l'information ne se retrouve pas chez Flodoard et sa source n'a pas été identifiée.

51. Lieu-dit «Le prieuré» (carte de l'I.G.N., 1/25000, 2511 OT). Autour de Paris, nombreuses furent les communautés religieuses qui, à partir de la fin du IX siècle, eurent pour refuge un castellum installé dans un secteur inondable. Le castrum de Pierrepont (Aisne), construit par l'évêque de Laon Didon († c. 895) pour servir d'abri à ses chanoines et à leurs reliques, était situé au milieu des marais (BuR, Michel, Vestiges d'habitat seigneurial fortifié des Ardennes et de la vallée de l'Aisne, II, Cahier des Lettres et Sciences Humaines de l'Université de Reims, n² 2, 1980, p. 97-99). Il en était de même du castrum de Palluau au confluent de la Juine et de l'Essonne (Dép. Essonne, cant. Mennecy, com. Ballancourt-sur-Essonne), où un groupe de clercs de l'église de Bayeux trouva asile vers le début des années 920 (LAIR, Jules, Etudes sur les origines de l'évêché de Bayeux, II, Bibliothèque de l'École 
La nouvelle source que constitue pour les historiens de la Croix-Saint-Ouen la lettre de Gautier d'Orléans vient ainsi compléter de manière particulièrement heureuse les informations des diplômes de 884 et de 918 , deux actes qui faisaient déjà de ce monastère le mieux documenté de la province de Rouen pour la fin du IX et le début du $\mathrm{X}^{\mathrm{e}}$ siècle. En dépit de sa brièveté, elle nous livre plusieurs éléments intéressants pour l'étude de cette période-clé de l'histoire de la future Normandie. À l'encontre de la thèse soutenue au cours de ces dernières années par plusieurs chercheurs américains, selon laquelle les raids scandinaves en Francie auraient été sans grandes conséquences pour les communautés religieuses ${ }^{52}$, elle apporte, si besoin en était encore, une preuve de la réalité des exodes monastiques. En présence d'un récit de translation de reliques, on est certes en droit, quelquefois, de suspecter le narrateur de faire œuvre de propagande ou de réécrire l'histoire à sa façon, d'autant que les récits de ce type ont souvent été rédigés bien après les événements. Il n'en est pas de même pour un document épistolaire comme celui-ci : écrit sur le champ pour régler un problème purement pratique - assurer le gîte et le couvert au groupe de réfugiés -, ce bref épiscopal a la valeur d'un témoignage pris sur le vif. Son intérêt est d'autant plus grand que les récits de voyages de corps saints nous parlent très rarement des religieux eux-mêmes et des conditions matérielles dans lesquelles ces communautés vivaient leur exode. Autre élément non négligeable pour l'analyse de la situation dans le secteur de la basse Seine à la fin du IX ${ }^{\mathrm{e}}$ siècle, la lettre de Gautier prouve que l'évacuation du monastère de la Croix-Saint-Ouen eut lieu au plus tard à la fin de l'année 885 , ce qui situe l'évènement à une date assez antérieure à celle de 898 avancée par Mabillon. Enfin, le nouvel éclairage qu'elle nous apporte sur les derniers temps du monastère montre que cet établissement fonctionnait encore de manière autonome au début du siège de Paris. Nous y voyons une raison de plus pour douter du bien-fondé de la thèse d'un monastère de la Croix placé dès le milieu du IX siècle sous la tutelle de Saint-Germain-des-Prés ${ }^{53}$. Au demeurant, il n'existe à ce jour aucune preuve de relations entre les deux établissements avant 918, date à laquelle Charles-le-Simple fit don à l'abbaye parisienne des corps saints et du reliquat de la mense conventuelle de la Croix-Saint-Ouen.

des Chartes, t. 24, 1863, p. 297); voir également CORVISIER, Élisabeth, «L'exode et l'implantation des reliques des saints de l'ouest de la France en Ile-de-France aux $\mathrm{IX}^{\mathrm{e}}$ et $\mathrm{X}^{\mathrm{e}}$ siècles ", Paris et Ilede-France, Mémoires, t. 32 (1981), p. 292.

52. Cf. notamment LiFSHITZ, Felice, "The exodus of Holy Bodies reconsidered; the date of the translation of Gildardus de Rouen from Rouen to Soissons », Analecta Bollandiana, t. 110 (1992), p. 329340 ; PotTs, Cassandra, «When the Saints Go Marching: Religious Connections and the Political Culture of Early Normandy ", in Anglo-Norman Political Culture and the 12 th-Century Renaissance, Warren Hollister Charles (éd.), Woodbridge, The Boydell Press, 1997, p. 28.

53. Sur nos réserves au sujet de cette théorie, voir ci-dessus, note 12. 


\section{Orléans, 885-890}

Lettre de Gautier, évêque d'Orléans, à Lambert, évêque du Mans, au sujet d'un groupe de moines de la Croix-Saint-Ouen qu'il a accueilli à Orléans et qui s'apprête à regagner le comté de Bayeux en passant par le diocèse du Mans.

$B$ Copie du IX ${ }^{\mathrm{e}}$ siècle dans BnF, nouv. acq. lat. 1096, fol. 95.

BISCHOFF, Bernhard, «Briefe des neunten Jahrhunderts", in Anecdota novissima. Texte der vierten bis sechzehten Jahrhunderts, Bernhard BISCHOFF (éd.), Quellen und Untersuchungen zur lateinischen Philologie, 7, Stuttgart, 1984, lettre n 1, p. 126-127.

La rédaction de cette lettre se place entre 883 et 890, dates de l'épiscopat de Lambert. On peut préciser qu'elle n'est sans doute pas antérieure à la fin de l'année 885 , qui correspond au début du siège de Paris par les Normands.

Reverentissimo atque excellentissimo L. episcopo G. humilis divina suffragante gratia Aurelianorum episcopus fidele (?) servicium cum........is orationibus. Vestram humiliter exposcimus reverentiam, ut nos et ecclesiam regimini nostro collatam vestris opifici rerum commendetis precibus. Prout enim plus gravi peccatorum premimur pondere, tanto magis vestris sacrosanctis indigemus oratonibus credentes (?) multum veniae promereri continuis vestrae caritatis supplicationibus. Nostra vero pro vobis vestrisque omnibus piissimam Christi clementiam exorare non desistit humilitas, ut vos finetenus inter varias labilis saeculi huius procellas almificus dignetur protegere Christus. Vestrae sublimi innotescere dignum duximus prudentiae, quia monachi de Cru (ce) sancti Audoeni dira Normannice persecutionis cogente crudelitate ad nostrae humilitatis confugere praesidium, quibus, prout contulit possibilitas, aliquantulum largiti sumus supplementum. Nunc ergo deficientibus eis victualibus sumptibus per vestrum episcopatum Baiocensem comitatum, ubi rerum suarum aliqua habetur fiducia, in praesenti eos adire cogit necessitas. Unde piam sanctitatis vestre exoramus paternitatem, ut eos pro eterni remuneratoris amore in clementissimo pietatis vestre gremio suscipientes utpote viris religiose Deo militantibus necessarium exhibere dignemini adiutorium. 